\title{
A randomized trial to assess the effect of cricoid displacing maneuver on the success rate of blind placement of double-lumen tube and Univent bronchial blocker
}

\author{
Zhao Xu ${ }^{1}$, Hong Yu ${ }^{1}$, Yuting $\mathrm{Luo}^{2}$, Yuancai Ye ${ }^{1}$, Cheng Zhou ${ }^{1,3}$, Peng Liang ${ }^{1}$ \\ ${ }^{1}$ Department of Anesthesiology, West China Hospital, Sichuan University, Chengdu, China; ${ }^{2}$ West China School of Medicine, Sichuan University, \\ Chengdu, China; ${ }^{3}$ Lab of Anesthesia \& Critical Care Medicine, Translational Neuroscience Center, West China Hospital of Sichuan University, \\ Chengdu, China \\ Contributions: (I) Conception and design: P Liang, C Zhou; (II) Administrative support: P Liang; (III) Provision of study materials or patients: Y Ye, P \\ Liang; (IV) Collection and assembly of data: Z Xu, H Yu, Y Luo; (V) Data analysis and interpretation: Z Xu, H Yu, P Liang; (VI) Manuscript writing: \\ All authors; (VII) Final approval of manuscript: All authors. \\ Correspondence to: Peng Liang. Department of Anesthesiology, West China Hospital, Sichuan University, No.37 Guoxuexiang, Chengdu 610041, \\ China. Email: liangpeng@wchscu.cn.
}

Background Double lumen tube (DLT) and Univent are two commonly used lung isolation devices
that often require bronchoscopy assistance. In order to facilitate blind placement for situations where
bronchoscopy was unavailable, the cricoid displacing maneuver (CDM) was adopted. This study was
designed to explore whether the CDM could improve the successful blind placement rate in left lung
isolation esophageal surgeries.

Methods: One hundred and twenty ASA 1-3 patients who received left lung isolation esophageal surgeries from October 2014 to February 2016 and October 2018 to January 2020 were enrolled in this singlecentered prospective assessor-blinded randomized controlled trial. After anesthesia induction, patients were intubated either with DLT or Univent by applying the CDM, and the position of devices was checked by bronchoscopy. After turning into the right decubitus position, the devices were pulled back to the trachea, and placement was conducted once again. Successful placement rate for the first attempt, time needed for initial placement, malposition and reposition times by bronchoscopy during surgery, bronchus injury score, rank of lung isolation and postoperative anesthesia-related complications were recorded.

Results: At supine position, the successful placement rates for the first attempt of DLT-CDM, DLTcontrol, Univent-CDM and Univent-Control were 100\%, 76.7\%, 96.7\% and $66.7 \%$ respectively (DLT: $\mathrm{P}=0.016$; Univent: $\mathrm{P}=0.003$ ) while at right decubitus position were $86.7 \%, 66.7 \%, 93.3 \%$ and $66.7 \%$ (DLT: $\mathrm{P}=0.067$; Univent: $\mathrm{P}=0.010)$. There was no significant difference in the time needed for successful intubation and no severe complications.

Conclusions: The current study provided evidence that the CDM is an effective and safe blind endobronchial placement technique for left DLT and Univent. We suggest that CDM could be applied to the endobronchial placement of left DLT and Univent.

Keywords: Cricoid displacing maneuver; double-lumen tube; Univent; blind placement; complication

Submitted Oct 20, 2020. Accepted for publication Nov 12, 2020.

doi: 10.21037/apm-20-2065

View this article at: http://dx.doi.org/10.21037/apm-20-2065 


\section{Introduction}

Lung isolation techniques are conducted when it is necessary to ventilate one lung for surgical exposure. One lung ventilation (OLV) has become a standard approach and is increasingly being used for pulmonary, thoracic, mediastinal, vascular, esophageal, and orthopedic spine procedures $(1,2)$. OLV could also prevent contamination from the contralateral lung. OLV is mostly achieved by using conventional double-lumen tubes (DLT). Other alternative devices commonly used include various blockers (3).

The placement of DLT and Univent are relatively fast and malposition during surgery is uncommon, but correct placement can be difficult and even impossible in some extreme cases. During the blind placement process of either left DLT or Univent bronchial blocker, the device could be malpositioned into the right bronchus. In order to facilitate the correct placement rate, Fukuyama et al. introduced a blind left endobronchial placement technique for Univent in an article published in 2003. After turning the patient's head left, the operator places the right hand on the cricoid cartilage, and then press downward and towards the right to displace the larynx of the patient. In this case, the airway forms a left curve that could guide the blocker toward the left main bronchus (4) (Figure 1).

To our knowledge, there were no prospective randomized clinical trials assessing the efficacy of this maneuver in the placement of left DLT and Univent. We hypothesized that this maneuver would be a more suitable blind endobronchial placement method for left DLT and Univent. This prospective study was aimed to determine whether this cricoid displacing maneuver could facilitate the correct blind placement process of DLT and Univent.

We present the following article in accordance with the CONSORT reporting checklist (available at http://dx.doi. org/10.21037/apm-20-2065).

\section{Methods}

\section{Study design}

The study was conducted in accordance with the Declaration of Helsinki (as revised in 2013). The study was approved by the Ethics Committee of Clinical and Biomedical Trials, West China Hospital of Sichuan University (NO. 2014-90) and informed consent was taken from all individual participants. And registered in the Chinese Clinical Trial Register (ChiCTR) (ChiCTRIOC-14005313, http://www. chictr.org.cn/index.aspx). This

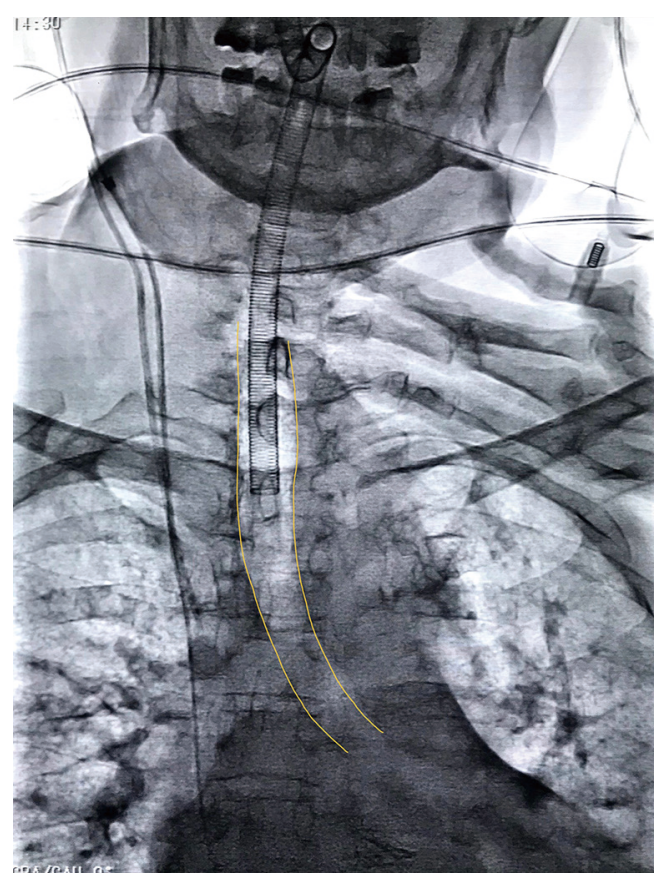

Figure 1 Fluoroscopy of the airway of a patient under cricoid displacing maneuver. The yellow lines suggest the changed alignment of the trachea and the left bronchus.

single center randomized controlled trial was conducted in the affiliated West China Hospital of Sichuan University and patients were enrolled according to the surgery planning list from October 2014 to February 2016 and October 2018 to January 2020. After acquiring a written consent form, 120 American Society of Anesthesiologists physical status 1-3 patients between 18 and 65 undergoing esophageal procedure requiring left thoracotomy were included, and the height of females should be more than $150 \mathrm{~cm}$ and male $160 \mathrm{~cm}$.

Exclusion criteria were as follows: Patients with anticipated difficult airway; BMI more than $28 \mathrm{~kg} / \mathrm{m}^{2}$; central bronchogenic carcinoma; pyothorax; bronchopleural fistula; and having difficulty in lung isolation.

Consecutive patients were randomly assigned to 4 groups: (I) the DLT-CDM group, (II) the DLT-control group, (III) the Univent-CDM group or (IV) the Univentcontrol group. Simple randomization was achieved using a table of random numbers originated from SPSS 18.0 by an independent investigator that were kept in sequentially numbered opaque envelopes. The randomization process was conducted immediately before the induction of anesthesia by the attending anesthesiologist in the OR. 


\section{Anesthesia procedure}

After routine fasting, all patients received a standardized intravenous premedication of $0.5 \mathrm{mg}$ Penehyclidine Hydrochloride Injection (Chengdu List Pharmaceutical Co., Ltd, China) as antisialagogue and $0.03 \mathrm{mg} / \mathrm{kg}$ midazolam for sedation 30 minutes before induction in the anesthesia preparing room. After entering the operating room, routine monitoring of electrocardiography, pulse oximetry, non-invasive arterial blood pressure, capnography, and body temperature were started. Then, after 3 minutes of preoxygenation, sufentanil $(0.3 \mu \mathrm{g} / \mathrm{kg})$, propofol $(2 \mathrm{mg} / \mathrm{kg})$ and rocuronium bromide $(0.6 \mathrm{mg} / \mathrm{kg})$ were administered intravenously for induction.

\section{Intubation procedure}

After induction of anesthesia, the resident anesthesiologist rotating in thoracic anesthesia less than 1 month with few experiences of DLT or Univent intubated the patients either with DLT or Univent by direct laryngoscopy while the attending anesthesiologist performed the cricoid displacing maneuver. Surgeons were absent from the operating room during intubation and were not aware of the device used.

Patients assigned to the DLT groups were intubated using a left-sided DLT (Broncho-cath, left-sided; Rusch, Kernen, Germany) of an adequate size according to left bronchus width measured on CT scan ( 35 or $37 \mathrm{Fr}$ ). The approximate depth of insertion from the incisor of DLT was calculated using the formula: DLT depth $(\mathrm{cm})=12.5+0.1$ $x$ height $(\mathrm{cm})(5)$. In the DLT-control group, after the tip of the endobronchial tube passing the vocal cords and the stylet removed, the left DLT was rotated 90 degrees to the left while being advanced. In the meantime, in the DLTCDM group, the DLT was placed according to Fukuyama's method described above.

Patients assigned to the Univent groups were intubated with a Univent tube (TCB type, Fuji Systems, Tokyo, Japan). Size 7.5 were used for male and 7.0 for female patients. The depth of the tube was about $23 \mathrm{~cm}$ for males and $21 \mathrm{~cm}$ for females. In the Univent-control group, after intubation of the main tube, the blocker was rotated 90 degrees while being advanced. The same as the DLT-CDM group, in the Univent-CDM group the blocker was placed according to Fukuyama's method.

Bronchoscopy examination was then conducted to determine the position of devices. If the endobronchial lumen of DLT or blocker of Univent was not in the right position, a second attempt by the same method was taken. For the control group, if two attempts both failed then a cricoid displacing maneuver was undertaken for the third attempt.

After turning into the right lateral decubitus position, the cuffs were loosened, and the devices were pulled back to the trachea to mimic the clinical situation of malposition. Auscultation was performed to confirm bilateral breath sounds. The placement was attempted following the same measures of each group. At this time, if the first attempt should fail, the cricoid displacing maneuver was performed for the second attempt. If both the attempts should fail, bronchoscopy was conducted.

The primary outcome of this study was the successful positioning rate for the first attempt. Other outcomes of our investigation included the time needed for initial placement, malposition and reposition times by bronchoscopy during surgery. The malposition of the device was determined by bronchoscopy. Malposition was described as follows: bronchial cuff herniated into carina, bronchial cuff edge not visible in the entrance of the mainstem bronchus, or double-lumen endotracheal tube, Univent blocker in the opposite bronchus. The time needed for initial placement was defined as the time from exposure of the vocal cords to placement of the endobronchial lumen in the supine position. While in the right lateral decubitus position, the time needed for initial placement was defined as the time from pulling back of the tube in the trachea to placement of the endobronchial lumen. Upon completion of the surgery, all the patients received a bronchoscopy examination before emergence from anesthesia for bronchus injury scoring which was classified as follows: 0 , no changes; 1 , redness; 2, edema; and 3, hematoma (6). At the end of the case, the surgeon was asked to give an overall assessment of lung isolation. Another important focus of our study was the postoperative anesthesia-related complications. Incidence of complications including laryngospasm, stridor, teeth damage, sore throat, and hoarseness was also documented 24 hours after surgery by a blinded investigator. The intensity of sore throat was defined as follows: 1, mild (pain with deglutition); 2, moderate (constant pain and exacerbation with deglutition); 3 , severe (pain interfering with eating and requiring analgesic) $(6,7)$.

\section{Statistical analysis}

All the data were blinded from the analyzer for statistical analysis. The continuous variables were presented as mean $\pm \mathrm{SD}$ or median with interquartile range. Categorical variables were presented as numbers (\%). Data were tested 


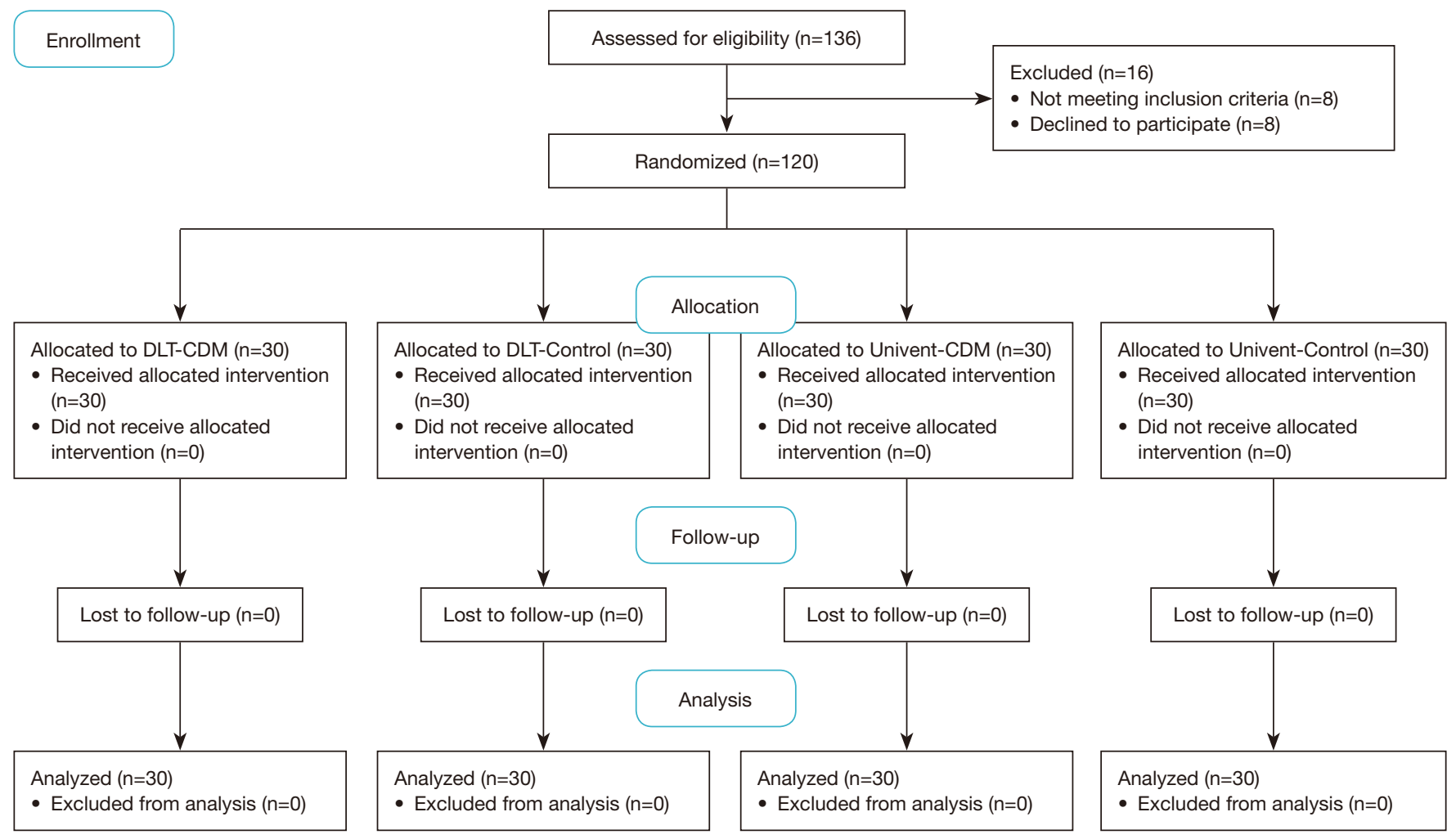

Figure 2 Flowchart of the study. DLT, double-lumen tube; CDM, cricoid displacing maneuver.

for normal distribution using the Kolmogorov-Smirnov test. A comparison of nonparametric data was performed using the Fisher exact test. Parametric data were analyzed using the unpaired student $t$-test. Ranking data was analyzed using the Mann-Whitney U test. A two-sided P value of less than 0.05 was considered statistically significant. All statistical analyses were performed by SPSS 18.0 for Windows (SPSS, Chicago, IL, USA).

The sample size was calculated based on the previous study which reported a successful first placement rate of $75.9 \%$ (2) and to allow the detection of a $30 \%$ difference in the success rate between CDM and control groups. Thus, based on the power of 0.8 and $\alpha$ error of $5 \%$, we estimated the sample size as 24 patients in each group. Because of the clinical setting and the potential risk of failure to placement despite comprehensive preparation, 30 patients were finally enrolled in the trial for each group.

\section{Results}

Initially, 136 consecutive patients in the study periods who underwent left thoracotomy requiring lung isolation were screened (Figure 2). Eight of them did not meet the inclusion criteria and eight refused to participate. A total of 120 patients were enrolled and randomized from October 2014 to February 2016 and October 2018 to January 2020. There were no significant differences in baseline characteristics between the corresponding groups (Table 1).

The successful placement rate in the supine position was significantly higher in the CDM group than the control group either for DLT (100\% vs. $76.7 \%, \mathrm{P}=0.016)$ or Univent $(96.7 \%$ vs. $66.7 \%, \mathrm{P}=0.003)$. There was no significant difference in the successful placement rate of DLT in the right lateral decubitus position between CDM and control groups $(86.7 \%$ vs. $66.7 \%, \mathrm{P}=0.067)$ while it was significantly higher in the Univent-CDM group (93.3\% vs. $66.7 \%, \mathrm{P}=0.010$ ). Lung isolation ranked by the surgeons and reposition times by bronchoscopy during surgery also showed no significant difference. The placement time, no matter in the supine position or in the right lateral decubitus position, was not significantly prolonged compared to the control group for both devices (Table 2).

Considering the complications, there was no significant difference in bronchus injury score between corresponding 
Table 1 Baseline characteristics of the patients

\begin{tabular}{|c|c|c|c|c|}
\hline & \multicolumn{2}{|c|}{ Left DLT } & \multicolumn{2}{|c|}{ Univent bronchial blocker } \\
\hline Age $(\mathrm{yr})$, mean $[\mathrm{IQR}]$ & 55 [23-69] & $52[30-79]$ & 64 [53-78] & 59 [50-69] \\
\hline Gender (M/F) & $18 / 12$ & $16 / 14$ & $15 / 15$ & $17 / 13$ \\
\hline BMI $\left(\mathrm{kg} / \mathrm{m}^{2}\right)$, mean (SD) & $23.43(1.51)$ & $23.06(2.22)$ & $22.71(7.83)$ & $21.59(2.06)$ \\
\hline Mallampati class $(1 / 2 / 3 / 4)$ & $16 / 12 / 2 / 0$ & $15 / 13 / 2 / 0$ & $18 / 11 / 1 / 0$ & $17 / 12 / 1 / 0$ \\
\hline $\begin{array}{l}\text { Tube type (DLT: 37\#/35\#); } \\
\text { (Univent: } 7.5 \# / 7.0 \#)\end{array}$ & $17 / 13$ & $16 / 14$ & $15 / 15$ & $17 / 13$ \\
\hline $\begin{array}{l}\text { Surgical approach (open/ } \\
\text { thoracoscopy) }\end{array}$ & $12 / 18$ & $10 / 20$ & $9 / 21$ & $11 / 19$ \\
\hline
\end{tabular}

DLT, double-lumen tube; CDM, cricoid displacing maneuver; IQR, interquartile range; BMI, body mass index; SD, standard deviation; ASA, American Society of Anesthesiologists.

Table 2 The effectiveness of cricoid displacing maneuver

\begin{tabular}{|c|c|c|c|c|c|c|}
\hline & \multicolumn{3}{|c|}{ Left DLT } & \multicolumn{3}{|c|}{ Univent bronchial blocker } \\
\hline \multicolumn{7}{|l|}{ Supine position } \\
\hline Successful placement at first attempt, n (\%) & $30(100.0)$ & $23(76.7)$ & 0.016 & $29(96.7)$ & $20(66.7)$ & 0.003 \\
\hline Successful placement at second attempt, $\mathrm{n}$ & 0 & 3 & - & 1 & 4 & - \\
\hline Time for initial placement at first attempt (s) & $128(22.8)$ & $122(18.1)$ & 0.264 & $154(42.2)$ & $158(22.8)$ & 0.650 \\
\hline \multicolumn{7}{|l|}{ Right lateral decubitus position } \\
\hline Successful placement at first attempt, n (\%) & $26(86.7)$ & $20(66.7)$ & 0.067 & $28(93.3)$ & $20(66.7)$ & 0.010 \\
\hline Time for initial placement at first attempt (s) & $75(28.7)$ & $79(31.2)$ & 0.607 & $85(11.9)$ & $81(22.5)$ & 0.393 \\
\hline Rank of surgical exposure (excellent/fair/bad) & $23 / 5 / 2$ & $24 / 3 / 3$ & 0.992 & $24 / 4 / 2$ & $26 / 3 / 1$ & 0.992 \\
\hline $\begin{array}{l}\text { Malposition and repositions by bronchoscopy } \\
\text { during surgery, } \mathrm{n}(\%)\end{array}$ & $5(16.7)$ & $3(10.0)$ & 0.448 & $6(20.0)$ & 5 (16.7) & 0.739 \\
\hline
\end{tabular}

DLT, double-lumen tube; CDM, cricoid displacing maneuver.

CDM and control groups. At the meantime, no incidences of severe tracheobronchial hemorrhage, tracheobronchial perforation, or arytenoid dislocation occurred in all the groups. There was no significant difference in the occurrence of sore throat and hoarseness. However, there was a significantly higher rate of postoperative sore throat in patients with DLT than with Univent (Table 3).

\section{Discussion}

In accordance with our previous study focusing on Arndt endobronchial blocker, the present study also demonstrated 
Table 3 The safety of cricoid displacing maneuver

\begin{tabular}{|c|c|c|c|c|c|c|}
\hline & \multicolumn{3}{|c|}{ Left DLT } & \multicolumn{3}{|c|}{ Univent bronchial blocker } \\
\hline Laryngospasm, n (\%) & $0(0)$ & $0(0)$ & - & $0(0)$ & $0(0)$ & - \\
\hline Sore throat, n (\%) & $10(33.3)$ & $15(50.0)$ & 0.190 & $5(16.7)$ & $5(16.7)^{\star}$ & 1.000 \\
\hline Intensity of sore throat $(1 / 2 / 3)$ & $5 / 2 / 3$ & $8 / 5 / 2$ & 0.674 & $3 / 2 / 0$ & $2 / 1 / 2$ & 0.401 \\
\hline Hoarseness, n (\%) & $2(6.7)$ & $3(10)$ & 0.640 & $1(3.3)$ & $2(6.7)$ & 0.554 \\
\hline
\end{tabular}

${ }^{*} \mathrm{P}=0.001$, compared between pooled DLT and Univent data using Fisher's exact test. DLT, double-lumen tube; CDM, cricoid displacing maneuver; SD, standard deviation.

that applying CDM could elevate the successful placement rate of DLT and Univent (8). Combining with CDM, the successful placement rate in the supine position for the first attempt could be up to $100 \%$ for DLT and $96.7 \%$ for Univent. In the right lateral decubitus position, the rates declined to $86.7 \%$ and $93.3 \%$ for DLT and Univent respectively. Despite the additional step of applying force to the cricoid cartilage, the overall intubation time was not significantly elongated compared to the control group for both devices.

DLT has been generally accepted as a standard technique for lung isolation during thoracic surgeries because it could be positioned more quickly, less likely to malposition, and its accessibility of the non-ventilated lung (2,9-11). Considering the placement of DLT, it is more difficult in patients with abnormal airway anatomy especially for right DLT and difficult airways (8,12-14). Although Univent presented quite a few advantages, there were still some studies argued that Univent might have no advantage over DLT (15-19). In 2007, Campos et al. rigorously discussed the disadvantages of bronchial blockers (9). A distinct one was that the blocker could be easily misplaced into the right bronchus. Another obvious disadvantage was the intermittent requirement of bronchoscopy even though it is generally accepted as the gold standard. Certain bronchoscopes have irrigation and suction functions, which further facilitated the placement process $(9,20)$. However, not all anesthesia departments are equipped with bronchoscopes, especially in some rural areas of China. Other than that, it was not only time-consuming but also labor-intensive, and the learning curve was relatively steep (21-23). According to multiple previous studies, the incidence of malposition of bronchial blockers during surgery was higher than that of DLTs and it was not easy to place the bronchial blocker into the left main bronchus using method recommended by the manufacturer (24-27). In the present study, the CDM increased the success rate compared with previous studies $(28,29)$. As aforementioned, the force applied to the larynges could align the left main bronchus with the trachea (Figure 1). Therefore, as predicted the endobronchial part of the devices could be easily slided down to the targeted bronchus. Although the success rate reduced in the lateral decubitus position, the CDM group still presented with a higher rate. This could be related to the special anatomy in the right lateral decubitus position and the tube might be more difficult to insert into the left bronchus due to gravity. In addition, even if an additional step of CDM was applied, the overall time needed for initial placement for Univent was reduced compared with previous Light stylet guided placement described by Weng et al. which consumed the least time $(154 \pm 42.2$ vs. $176 \pm 50 \mathrm{~s}, \mathrm{P}=0.1486)$ (22). The application of $\mathrm{CDM}$ reduced the time compared with multiple previous studies $(158-804$ s) $(25,27,28)$. These results all suggest that combining CDM, the placement of DLT and Univent could be less time-consuming and more efficient.

As for complications, many researchers have reported the side effects of DLT and Univent, especially sore throat and hoarseness that could cause medical disputes. Since the cricoid manipulating maneuver affected the airway 
structure, it could potentially induce more postoperative complications. In the present study, the bronchus injury score and incidence of postoperative complications were similar between the corresponding groups. The maneuver did not induce elevated risk of complications. As far as we know, there were no previous studies on the CDM reported injury to the trachea. In accordance with the previous study, a higher incidence of sore throat and hoarseness was observed in the DLT groups though (30). This phenomenon could suggest that the complications were mainly caused by the devices themselves, but not by the cricoid manipulating maneuver. These results all suggested that CDM could be applied to patients without causing severe complications. However, the force applied to the larynx could not be measured directly. In the present study, all CDM were performed by experienced attending anesthesiologists with caution. We suggest that in future applications, the person performing CDM should be gentle.

As for difficult airways, not long-ago Heir et al. reported two successful intubations in difficult airways with this maneuver. Although the placement was achieved with the assistance of advanced equipment (bronchoscopy and single lumen tube with an embedded distal tip camera), the external tracheal manipulating maneuver described by the authors played an important part in the placement of the blockers (23). By a combination of CDM and other advanced intubation equipment, placement of DLT and Univent could be achieved for difficult airways.

There are some limitations to this study. First, the sample size of this randomized trial was relatively small, and it was a single center study. The generalization of the results was limited. The study was conducted in two different time periods due to the schedule of the primary investigator. This might affect the results because of the advancement of perioperative management. Second, the bronchus injury score could be affected by the surgery itself or reposition during surgery. The interpretation of it should be cautious. Third, the equipment used in this study was limited to left DLT of 35 and $37 \mathrm{Fr}$ due to the supply of our department. Thus, height was set as an exclusion criterion for this study. Fourth, we only focused on patients with BMI less than $28 \mathrm{~kg} / \mathrm{m}^{2}$, the application of CDM on overweight and obese patients should be further validated. Last, we only compared CDM on left DLT and Univent, although it could be speculated that CDM could be applied to the placement process of right DLT and blockers, it should be validated in future studies to fully evaluate the safety and efficacy.
In conclusion, the cricoid manipulating maneuver is an effective and safe blind endobronchial placement technique of left DLT and Univent in the studied population. The cricoid displacing maneuver could facilitate the blind placement process of DLT and Univent blocker into the targeted bronchus with no increased side effects. We suggest that CDM could be applied to the endobronchial placement process of left DLT and Univent.

\section{Acknowledgments}

Funding: This work was supported by China National Natural Science Foundation (Grant No.81600918).

\section{Footnote}

Reporting Checklist: The authors have completed the CONSORT reporting checklist. Available at http://dx.doi. org/10.21037/apm-20-2065

Data Sharing Statement: Available at http://dx.doi. org/10.21037/apm-20-2065

Conflicts of Interest: All authors have completed the ICMJE uniform disclosure form (available at http://dx.doi. org/10.21037/apm-20-2065). The authors have no conflicts of interest to declare.

Ethical Statement: The authors are accountable for all aspects of the work in ensuring that questions related to the accuracy or integrity of any part of the work are appropriately investigated and resolved. The study was conducted in accordance with the Declaration of Helsinki (as revised in 2013). The study was approved by the Ethics Committee of Clinical and Biomedical Trials, West China Hospital of Sichuan University (NO. 2014-90) and informed consent was taken from all individual participants.

Open Access Statement: This is an Open Access article distributed in accordance with the Creative Commons Attribution-NonCommercial-NoDerivs 4.0 International License (CC BY-NC-ND 4.0), which permits the noncommercial replication and distribution of the article with the strict proviso that no changes or edits are made and the original work is properly cited (including links to both the formal publication through the relevant DOI and the license). See: https://creativecommons.org/licenses/by-nc-nd/4.0/. 


\section{References}

1. Narayanaswamy M, McRae K, Slinger P, et al. Choosing a lung isolation device for thoracic surgery: a randomized trial of three bronchial blockers versus double-lumen tubes. Anesth Analg 2009;108:1097-101.

2. Brodsky JB, Lemmens HJM. Left double-lumen tubes: clinical experience with 1,170 patients. J Cardiothorac Vasc Anesth 2003;17:289-98.

3. Wald SH, Mahajan A, Kaplan MB, et al. Experience with the Arndt paediatric bronchial blocker. Br J Anaesth 2005;94:92-4.

4. Fukuyama H, Nishiyama J, Kanazawa M, et al. Blind endobronchial insertion of a movable bronchial blocker attached to an endotracheal tube, Univent tube. Tokai J Exp Clin Med 2003;28:161-5.

5. Takita K, Morimoto Y, Kemmotsu O. The height-based formula for prediction of left-sided double-lumen tracheal tube depth. J Cardiothorac Vasc Anesth 2003;17:412-3.

6. Knoll H, Ziegeler S. Airway Injuries after One-lung Ventilation: A Comparison between Double-lumen Tube and Endobronchial Blocker: A Randomized, Prospective, Controlled Trial. Anesthesiology 2006;105:471-7.

7. McHardy FE, Chung F. Postoperative sore throat: cause, prevention and treatment. Anaesthesia 1999;54:444-53.

8. Liang P, Ni J, Zhou C, et al. Efficacy of a New Blind Insertion Technique of Arndt Endobronchial Blocker for Lung Isolation: Comparison With Conventional Bronchoscope-Guided Insertion Technique-A Pilot Study. Medicine (Baltimore) 2016;95:e3687.

9. Campos JH. Which device should be considered the best for lung isolation: double-lumen endotracheal tube versus bronchial blockers. Curr Opin Anaesthesiol 2007;20:27-31.

10. Campos JH. Current techniques for perioperative lung isolation in adults. Anesthesiology 2002;97:1295-301.

11. Clayton-Smith A, Bennett K, Alston RP, et al. A Comparison of the Efficacy and Adverse Effects of Double-Lumen Endobronchial Tubes and Bronchial Blockers in Thoracic Surgery: A Systematic Review and Meta-analysis of Randomized Controlled Trials. J Cardiothorac Vasc Anesth 2015;29:955-66.

12. Alsharani H, Eldawlatly A. Lung isolation algorithm: A novel template. Saudi J Anaesth 2014;8:447-8.

13. Campos JH. Lung isolation techniques for patients with difficult airway. Curr Opin Anaesthesiol 2010;23:12-7.

14. de Bellis M, Accardo R, Di Maio M, et al. Is flexible bronchoscopy necessary to confirm the position of doublelumen tubes before thoracic surgery? Eur J Cardiothorac
Surg 2011;40:912-6.

15. Takenaka I, Aoyama K, Kadoya T. Use of the univent bronchial-blocker tube for unanticipated difficult endotracheal intubation. Anesthesiology 2000;93:590-1.

16. Benumof JL, Gaughan S, Ozaki GT. Operative lung constant positive airway pressure with the Univent bronchial blocker tube. Anesth Analg 1992;74:406-10.

17. MacGillivray RG. Evaluation of a new tracheal tube with a movable bronchus blocker. Anaesthesia 1988;43:687-9.

18. Kim Y. Positioning of the univent tube with bronchial blocker without fibreoptic bronchoscopy. Eur J Anaesthesiol 2007;24:642-3.

19. Inoue H, Shohtsu A, Ogawa J, et al. New device for onelung anesthesia: endotracheal tube with movable blocker. J Thorac Cardiovasc Surg 1982;83:940-1.

20. Slinger P. Con: the new bronchial blockers are not preferable to double-lumen tubes for lung isolation. J Cardiothorac Vasc Anesth 2008;22:925-9.

21. Grum DF, Porembka D. Misconceptions regarding double-lumen tubes and bronchoscopy. Anesthesiology 1988;68:826-8.

22. Brodsky JB. Placement of double lumen tubes--time to shed light on an old problem. Br J Anaesth 2000;85:166-7.

23. Heir JS, Sekhon AK, Thakar DR, et al. External Tracheal Manipulation Maneuver (ETMM) to Facilitate Endobronchial Blocker Placement. J Cardiothorac Vasc Anesth 2016;30:1061-3.

24. Campos JH, Reasoner DK, Moyers JR. Comparison of a modified double-lumen endotracheal tube with a singlelumen tube with enclosed bronchial blocker. Anesth Analg 1996;83:1268-72.

25. Campos JH, Kernstine KH. A comparison of a left-sided Broncho-Cath with the torque control blocker univent and the wire-guided blocker. Anesth Analg 2003;96:283-9, table of contents.

26. Zheng H, Duan Y, Geng W-M, et al. A comparison of double-lumen endotracheal tube with univent blocker and bronchial blocker during thoracic surgical anesthesia. Zhonghua Yi Xue Za Zhi 2012;92:2481-4.

27. Weng H, Xu ZY, Liu J, et al. Placement of the Univent tube without fiberoptic bronchoscope assistance. Anesth Analg 2010;110:508-14.

28. Campos JH, Hallam EA, Van Natta T, et al. Devices for lung isolation used by anesthesiologists with limited thoracic experience: comparison of double-lumen endotracheal tube, Univent torque control blocker, and Arndt wire-guided endobronchial blocker. Anesthesiology 2006;104:261-6, discussion 5A. 
29. Ruetzler K, Grubhofer G, Schmid W, et al. Randomized clinical trial comparing double-lumen tube and EZBlocker for single-lung ventilation. Br J Anaesth 2011;106:896-902.

Cite this article as: $\mathrm{Xu} \mathrm{Z,} \mathrm{Yu} \mathrm{H,} \mathrm{Luo} \mathrm{Y,} \mathrm{Ye} \mathrm{Y,} \mathrm{Zhou} \mathrm{C,} \mathrm{Liang}$ P. A randomized trial to assess the effect of cricoid displacing maneuver on the success rate of blind placement of doublelumen tube and Univent bronchial blocker. Ann Palliat Med 2021;10(2):1976-1984. doi: 10.21037/apm-20-2065
30. Zhong T, Wang W, Chen J, et al. Sore throat or hoarse voice with bronchial blockers or double-lumen tubes for lung isolation: a randomised, prospective trial. Anaesth Intensive Care 2009;37:441-6. 